Ann. Zootech., I964 13, (3). 24I-253.

\title{
EFFET DES TAUX ÉNERGÉTIQUE ET PROTIDIQUE DE LA RATION SUR L'EFFICACITÉ ALIMENTAIRE ET SUR LA CROISSANCE CHEZ LE PORCELET.
}

\author{
A. AUMAITRE, C. JOUANDET, E. SALMON-LEGAGNEUR \\ avec la collaboration technique de J. RetTagliati, M. Guichard et Huguette Dewulf \\ Station de Recherches sur l'Élevage des Porcs, \\ Centre national de Recherches zootechniques, Jouy-en-Josas (Seine-et-Oise)
}

\section{SOMMAIRE}

Au cours de deux expériences portant, l'une sur des porcelets sous la mère, de la naissance à $: 8$ semaines ( 28 portées), l'autre sur des porcelets sevrés à 5 semaines ( 12 portées), on a comparé l'efficacité de quatre régimes comportant deux taux de protéines et deux taux d'énergie, distribués suivant le dispositif factoriel $2 \times 2$.

Dans les deux expériences, les quantités d'aliment consommées diffèrent, mais la quantité d'énergie ingérée reste sensiblement la même dans tous les lots.

Chez le porcelet allaité, la vitesse de croissance et l'efficacité énergétique ne diffèrent pratiquement pas, seule l'efficacité protidique varie avec le taux de protéines de la ration.

Chez le porcelet sevré, les régimes qui provoquent les ingestions de protéines les plus élevées permettent la meilleure croissance, mais non les meilleurs efficacités. L'efficacité énergétique varie plus largement que l'efficacité protidique en fonction des taux respectifs d'énergie et de protéines de l'aliment.

Il en résulte que les régimes qui paraissent les plus avantageux (tant en ce qui concerne les vitesses de croissance que les efficacités alimentaires) diffèrent suivant qu'il s'agit de porcelets sevrés ou non. Un aliment pauvre en protéines et riche en énergie ( 13 P. 100 de protéines brutes, 4400 Calories brutes $/ \mathrm{kg}$ ) convient le mieux au porcelet allaité, alors qu'un aliment riche en protéines et d'une teneur moyenne en énergie ( $20 \mathrm{p}$. Ioo de protéines brutes, 4000 Calories brutes $/ \mathrm{kg}$ ) donne les meilleurs résultats chez le porcelet sevré à 8 semaines.

\section{IN'TRODUC'TION}

L"influence relative des taux énergétique et protidique de la ration sur la crois sance du jeune est connu depuis longtemps. MøLLGAARD (I929), LEROY (I937 et I954) expriment déjà les besoins en protéines d'un animal en fonction de la teneur en énergie 
de l'aliment. Plus récemment, de nombreux auteurs ont attiré l'attention sur l'importance du rapport $\begin{gathered}\text { Ǵnergie } \\ \text { Protéines }\end{gathered}$ lans l'alimentation des différentes espèces.

C'est ainsi que chez le Porc de 20 à roo kg, HARE et al. (I956) Pond et al. (Ig60), POIEY et SEERI.FY (I96I) WagNer et al. (I96I), Clawson et al. (I962), ont examiné divers aspects des relations entre les ingérés énergétiques et protidiques.

Par contre, on dispose de peu d'informations en ce qui concerne le porcelet allaité, ou en voie de sevrage aux environs de la $5^{\mathrm{e}}$ semaine ; on sait seulement toute l'importance de l'ingestion précoce d'aliment complémentaire sur la croissance du jeune porcelet (AUmatre et SAImon-LEgagneur, I96I). Celle-ci dépend en partie du choix des constituants du régime, mais on connait mal les aliments les mietux adaptés aux besoins énergétique et protidique du jeune porcelet.

Seuls PeO et al. (I957), SEWEi, et al. (I96I), LowREY et al. (I962) ont effectué quelques travaux sur ce sujet.

Dans le but d'apporter quelques précisions sur les taux énergétiques et protidiques des aliments pour porcelets, nous avons entrepris deux expériences portant, l'une sur des porcelets allaités de o à $\delta$ semaines, 1'autre sur des porcelets sevrés à 5 semaines (SAIMON-L,EGAGNELR et JouANier, I 962 ).

Dans les deux cas, 4 régimes alimentaires répondant aux normes les plus usuelles d'alimentation, mais différant légèrement quant aux taux d'azote et d'énergie, et à la nature des calories ont été comparés. Sans prétendre tirer des conclusions générales de ces expériences, nous avons voulu voir si de telles modifications étaient de nature à provoquer une variation des performances du jeune animal : gain de poids et efficacités alimentaires; pour certains animaux, l'adiposité en fin d'expérience a été également prise en considération.

\section{I. - EXPÉRIEXCE I PORCELETS ALLAITÉS}

\section{A. - M.ATÉRIEL LT MÉTHODES}

28 portées de porcelets de race Large If hite, élevés en porcherie conditionnée $\left(20^{\circ} \mathrm{C}, 60\right.$ p. Ioo d'humidité) de la naissance à 8 semaines, date de leur sevrage, étaient utilisées dans cette étude.

Ces portées étaient réparties en 4 lots, disposant à volonté pendant toute la période d'allaitement, de l'un des aliments granulés répondant aux caractéristirues générales suivantes:

- Miment e.t : tencur faible en énergie et élevéc en protéine.

- Aliment FA : teneur élevée en énergie et en protéine.

- Aliment ea : teneur faible en énergie et en protéine.

- Aliment Fa : teneur élevée en énergie et faible en protéine.

L'enrichissement en énergie résultait essentiellement d'une substitut ion d'une partie des glucides. du régime par des lipides. La composition des alinents est indiquée au tableau I.

Les productions laitières des truies étaient contrôlées et analysées (SALMON-LEGagNeUR, 1959) et il était tenu compte de l'apport protidique et énergétique du lait dans les calculs.

Pour chaque porcelet, on not ait le poids et le gain de poids et on calculait les quantités consommées d'aliment, d'énergie (mesurées ì la bombe calorimétrique à thermocouple de Féry), et de protéines ( $\mathrm{N}$ Kjeldahl $\times 6,25)$.

La signification des différences observées dans cette étude a été testée par l'analyse de variance appliquée au dispositif factoriel $2 \times 2$. 
TABI,EAU I

Composition des aliments complementaires

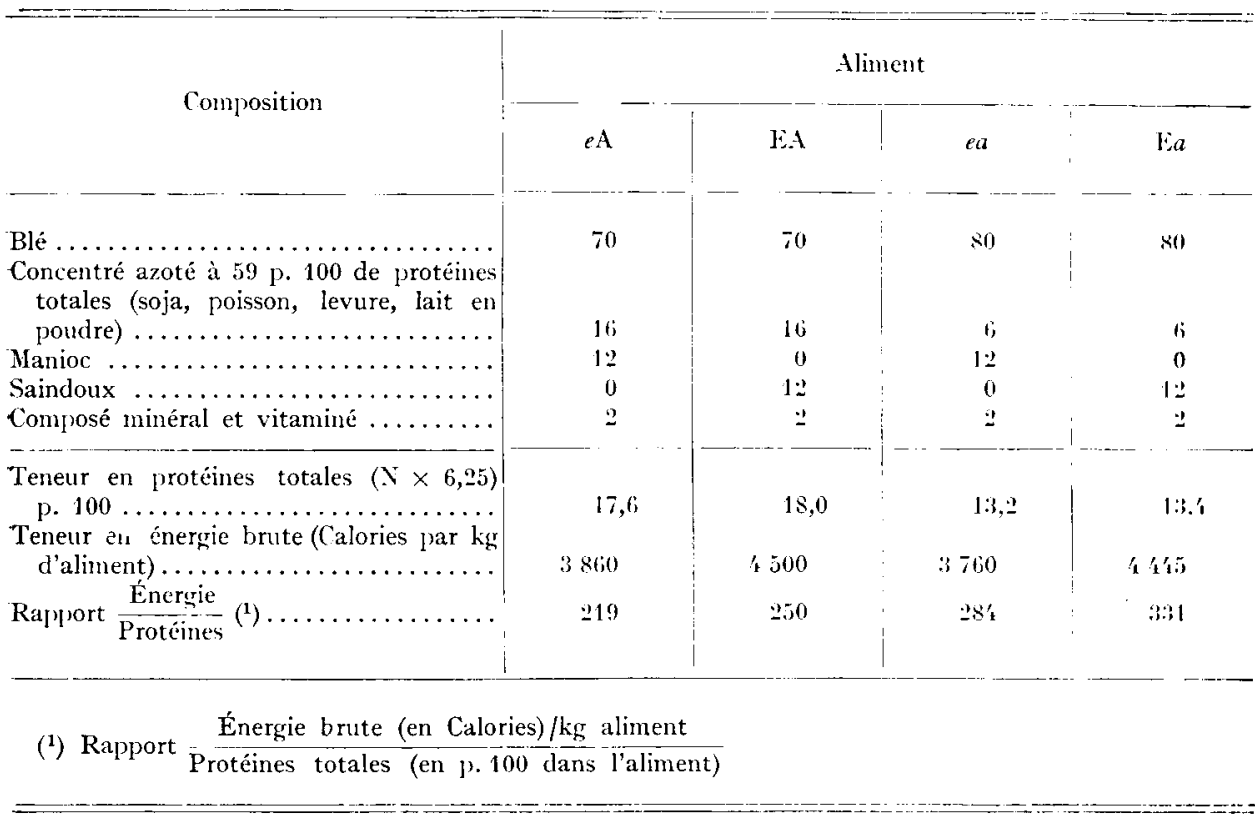

\section{B. - RÉSUITATS}

$\mathrm{I}^{\circ}$ Quantités consommées et gain de poids.

Le tableau 2 résume les principaux résultats concernant les poids moyens des porcelets de chaque lot et les quantités consommées de o à 8 semaines.

Les différents résultats permettent de faire les commentaires suivants : si les quantités de lait sont très voisines d'un lot à l'autre, (sauf en ce qui concerne le lot $\mathrm{E}(a)$, les quantités d'aliment consommées par porcelet semblent varier avec les lots.

On remarque en particulier que celles-ci ont été plus élevées pour les régimes qui présentaient la teneur en énergie la plus faible, ce que confirme l'effet « taux énergétique " trouvé par le calcul statistique.

Ces variations ont pour effet de maintenir à des niveaux voisins les quantités d'énergie et, à un degré moindre, les quantités de protéines consommées.

Dans la figure I, nous avons rapporté les ingérés protidiques et énergétiques entre la cinquième et la huitième semaine ainsi que les gains de poids pendant cette même période.

Les calculs effectués n'ont pas permis de mettre en évidence de différences significatives entre les différentes valeurs, mais l'examen de la figure I montre que les gains de poids se classent suivant les ingérés énergétiques plutôt que suivant les nigérés protidiques. 
A. AUMAITRE, C. JOUANDE'T, E. SALMON-LEGAGNEUR
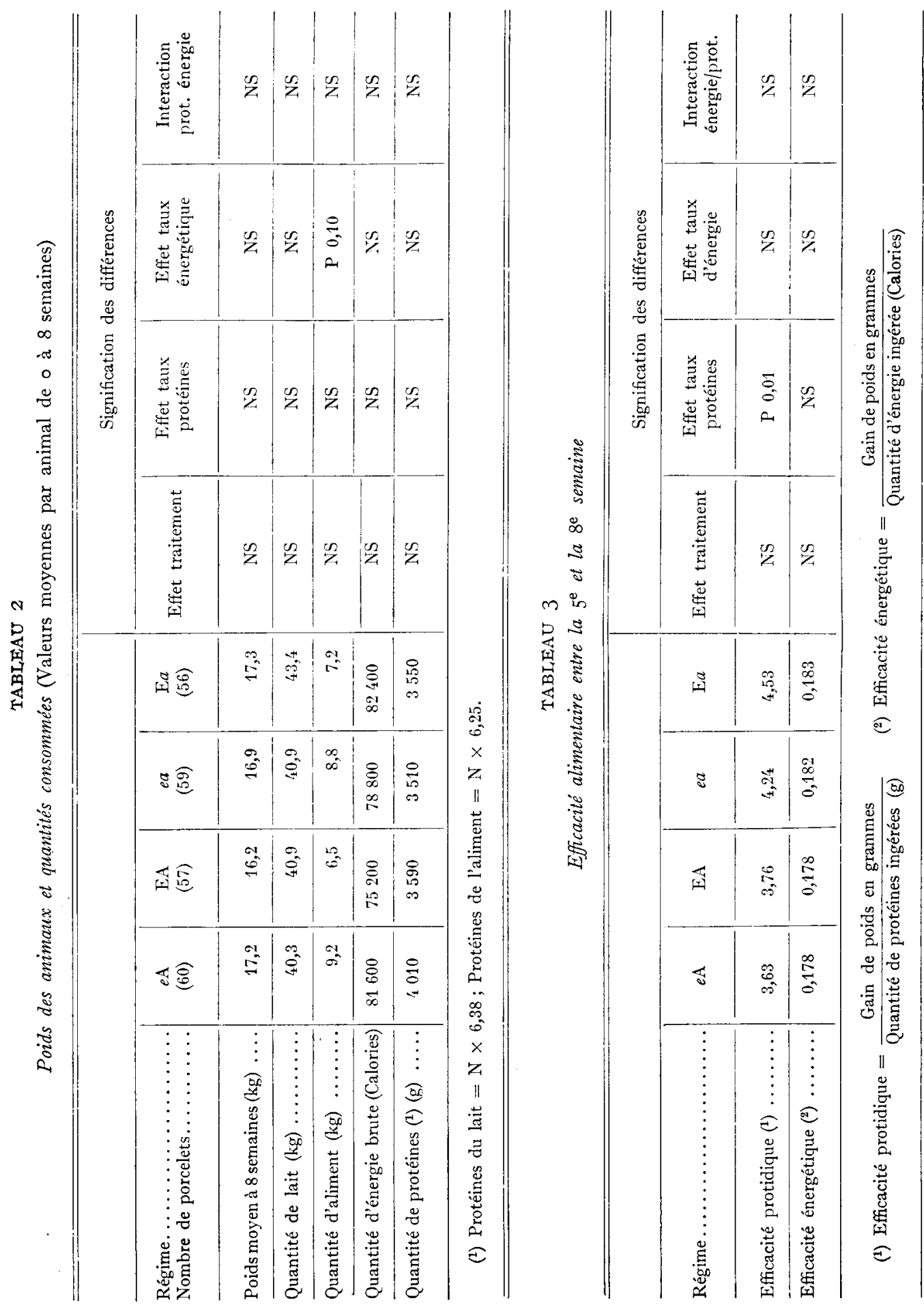


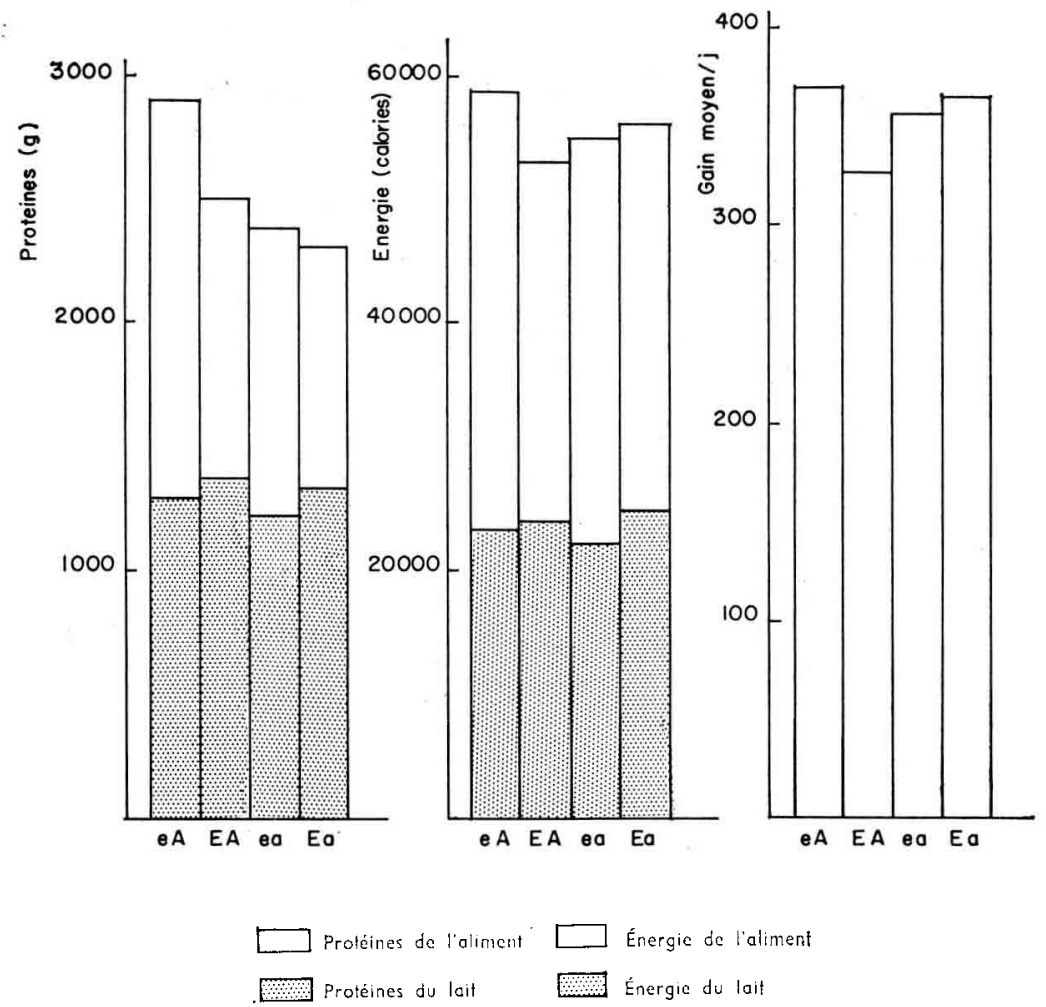

FIG. I. - Quantités de protéines el d'énergie ingérées par le porcelel allaité entre 5 et 8 semaines el gain moyen journalier

$2^{\circ}$ Efficacités alimentaires.

Il n'a pas été possible de dissocier les efficacités protidiques et énergétiques se rapportant au lait ou à l'aliment complémentaire. De ce fait, les calculs que nous avons effectués se rapportent aux efficacités de la ration totale (tabl. 3).

Une différence importante apparaît dans l'utilisation des protéines et de l'énergie : les efficacités énergétiques sont rigoureusement constantes quels que soient les lots, alors que les taux azotés élevés entraînent une diminution significative des efficacités protidiques.

$\left.3^{\circ}\right)$ Influence du rapport $\frac{\text { Énergie }}{\text { Protéines }}$ de l'aliment complémentaire.

La figure 2 retrace la variation du gain de poids moyen, des efficacités protidique et énergétique des ingérés selon les différents équilibres des aliments complémentaires utilisés.

On constate que le gain de poids est indépendant du rapport $\frac{\text { Énergie }}{\text { Protéines }}$ de l'aliment; il en va de même de l'efficacité énergétique. Seule l'efficacité protidique augmente en même temps que ce rapport. 


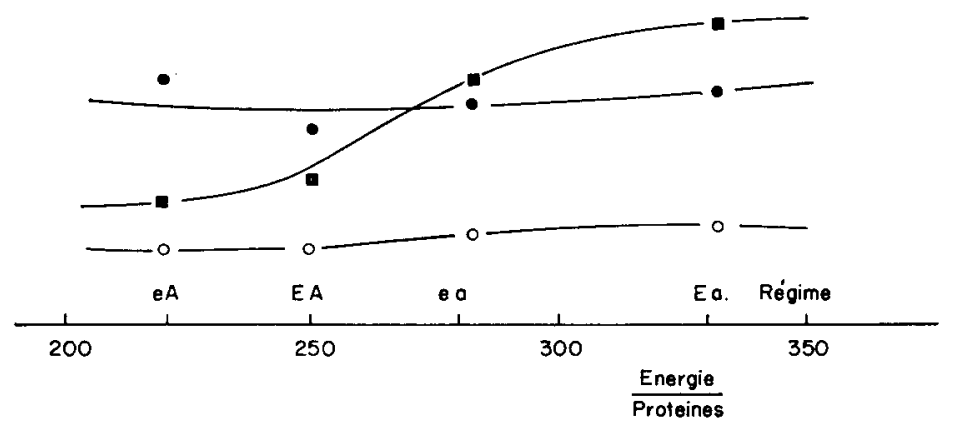

- Efficacité protidique

- Efficacité énergéfique

- Gain de poids

FIG. 2. - Variations alı gain de poids et des efficacités énergétiques el protidiques en fonction du rapport Energie

\section{II. - EXPÉRIENCE II : PORCELETS SEVRÉS A 5 SEMAINES}

\section{A. - MatéRIEL e'T Méthodes}

L'expérience a été effectuée sur 2 portées de porcelets de même provenance que précédemment.

Les animaux étaient sevrés à 5 semaines; au moment du sevrage, les portées étaient divisées en z groupes d'égale importance (nombre, poids, sexe) et affectés comme précédemment à deux des quatre traitements suivants : $e \mathrm{~A} ; \mathrm{EA} ; e a ; \mathrm{E} a$ (voir plus haut). La composition des régimes est indiquée au tableau 4.

A l'intérieur de chaque portée, les régimes étaient comparés 2 à 2 par permutation circulaire et on effectuait 2 répétitions de chacune des comparaisons.

Comme précédemment, on mesurait toutes les semaines la quantité d'aliment consommée par porcelet durant la période expérimentale et l'on déterminait les ingérés protidiques et énergétiques qui en résultaient. L'interprêtation statistique a été faite suivant la méthode des blocs incomplets équilibrés (Cochran, I962), ce qui a permis de tenir compte de l' " effet portée ». En outre, sur quelques portées, un essai d'appréciation de l'adiposité a été entrepris par mesure radiographique de l'épaisseur du tissu adipeux sous-cutané au niveau du cou, du dos et du rein suivant la technique de DUMONT et FÉVRIER (I957).

\section{B. - RÉsultats}

Io Quantités consommées et gain de poids.

Le tableau 5 rapporte les principaux résultats (moyennes par porcelet).

Comme pour les animaux sous la mère, on observe des différences importantes entre les quantités d'aliment consommées dans chaque groupe. Celles-ci résultent essentiellement de l'effet du taux énergétique des aliments et à un moindre degré du taux de protéines. Ces variations ont pour effet. de limiter les différences entre les quantités d'énergie consommées et de faire apparaître au contraire des différences en ce qui concerne les ingérés protidiques. 
Par ailleurs, les gains de poids sont significativement différents entre les lots : ils sont plus élevés pour les régimes à faible taux énergétique qui permettent une plus grande ingestion de protéines.

\section{TABLEAU 4}

Composition des aliments de sevrage à 5 semaines ( $p$. I00)

\begin{tabular}{|c|c|c|c|c|}
\hline \multirow{2}{*}{ Composition } & \multicolumn{4}{|c|}{ Aliment } \\
\hline & $e \mathrm{~A}$ & EA & $\varepsilon a$ & $\mathrm{E} a$ \\
\hline $\begin{array}{l}\text { Mélange de base à } 26 \mathrm{p} \cdot 100 \text { de protéines } \\
\text { totales (céréales } 61 \text {, soja } 6 \text {, poisson } 13, \\
\text { levure } 6 \text {, lait en poudre } 1 \text { 't } \ldots \ldots \ldots \ldots \text {. }\end{array}$ & 81 & 81 & 68 & 68 \\
\hline Amidon de mais...$\ldots \ldots \ldots \ldots \ldots$ & 7 & 0 & 17 & 6 \\
\hline 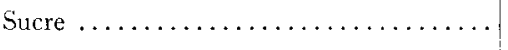 & 8 & 1 & 11 & 8 \\
\hline Saindoux ou huile d'arachice........ . & 1 & 15 & 1 & 15 \\
\hline Composé minéral et vitaminé...$\ldots \ldots$. & 2 & 2 & 2 & 2 \\
\hline Teneur en protéines totales (p. 100) .... & 20,6 & 20,7 & 17,5 & 17,5 \\
\hline $\begin{array}{l}\text { Teneur en énergie brute }: \text { Calories } / \mathrm{kg} \text { d'ali- } \\
\text { ment } \ldots \ldots \ldots \ldots \ldots \ldots \ldots \ldots \ldots \ldots \ldots\end{array}$ & 4020 & $46 ; 0$ & 4020 & 4510 \\
\hline Rapport $\underset{\text { Erónergie }}{\text { Próines }} \ldots \ldots \ldots \ldots \ldots \ldots$ & 197 & 227 & 231 & 266 \\
\hline
\end{tabular}

Par contre, il ne semble pas y avoir de relation définie entre la vitesse de croissance et 1'ingéré énergétique.

\section{0) Efficacité alimentaire.}

Le tableau 6 rapporte les valeurs moyennes obtenues de la $5^{\mathrm{e}}$ à la $8^{\mathrm{e}}$ semaine.

Les différences d'efficacité les plus importantes portent sur l'utilisation de l'énergie. Cette dernière est plus élevée avec les régimes pauvres en énergie, résultat parfaitement classique.

Par contre, les différences sont moins nettes en ce qui concerne les efficacités protidiques; elles sont dues aux taux protidiques des régimes plutôt qu'aux quantités consommées : les régimes les plus riches en azote présentent les efficacités les plus faibles.

$3^{\circ}$ Influence du rapport $\frac{\hat{E} \text { nergie }}{\text { Protéines }}$ de l'aliment.

La figure 3 représente pour les différents régimes les variations du gain journalier, des efficacités protidiques et énergétiques en fonction du rapport $\frac{\text { Énergie }}{\text { Protéines }}$ des aliments. 


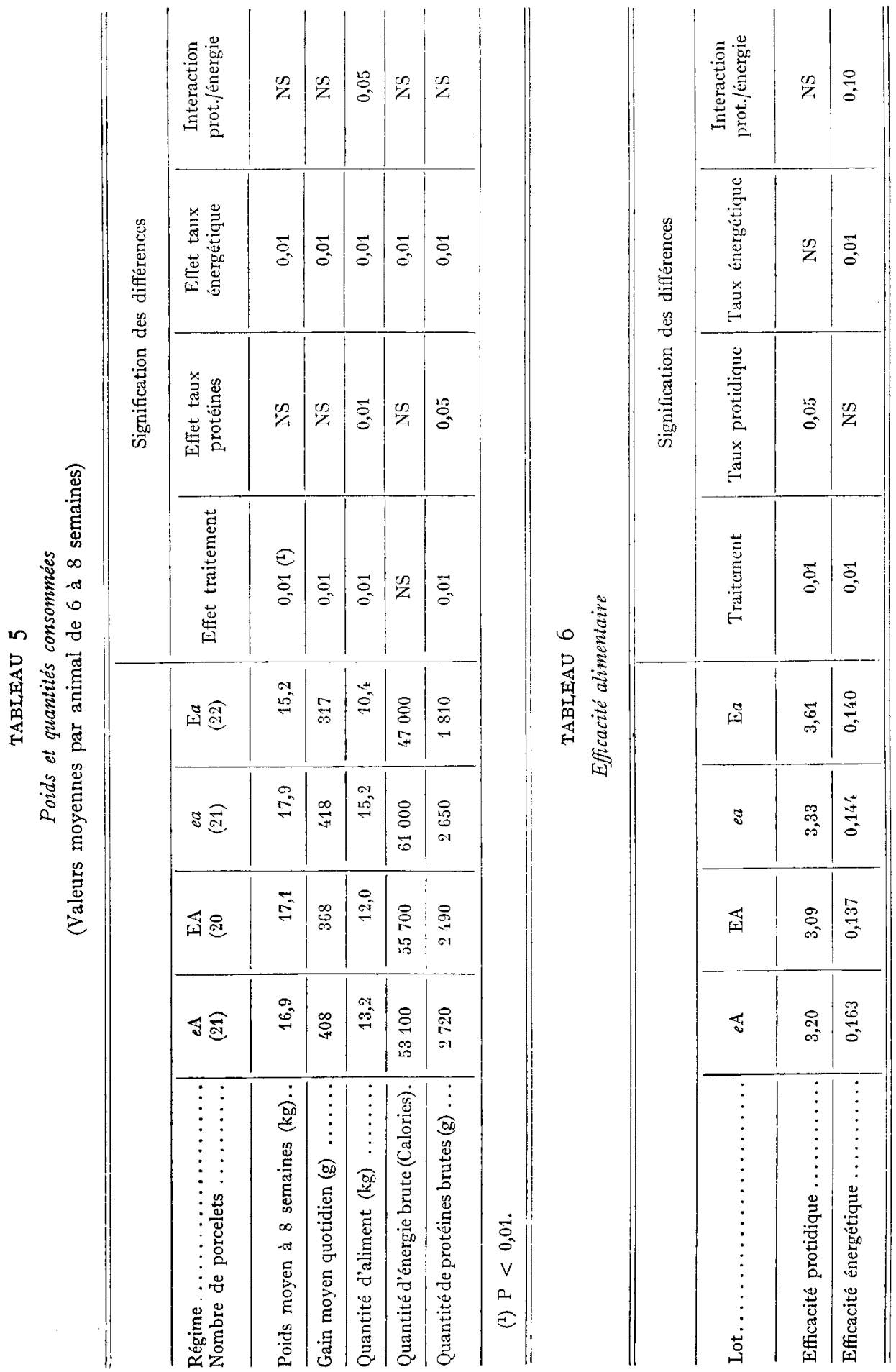


Malgré 1e nombre limité de nos observations, certaines tendances apparaissent:

- lorsque le rapport $\frac{\text { Énergie }}{\text { Protéines }}$ augmente, l'efficacité énergétique et le gain de poids diminuent ;

- dans les mêmes conditions, l'efficacité protidique semble augmenter.

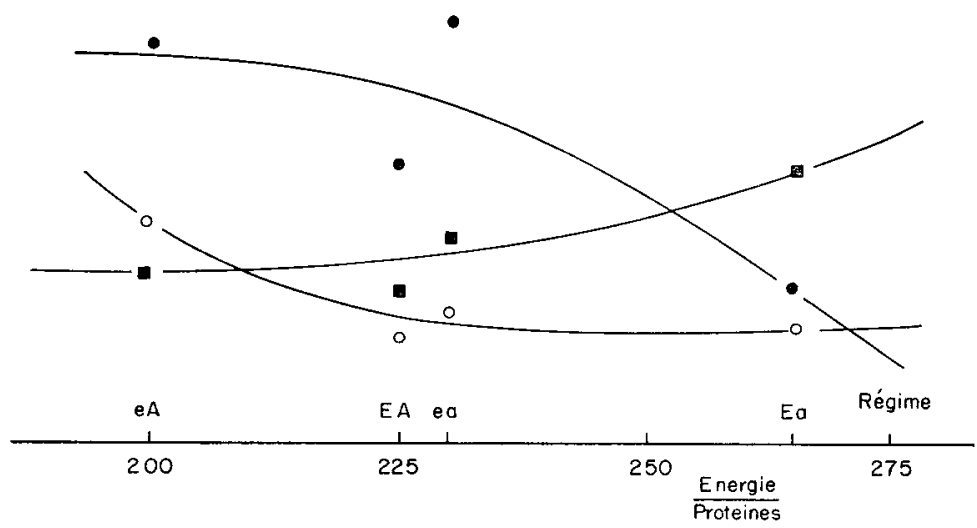

- Efficacité protidique

- Effiçacité énergétique

- Gain de poids

Fig. 3. - Variations du gain de poids et des eflicacités énergétiques et protidiques en fonction du rapport $\frac{\text { Energie }}{\text { Protéines }}$

$4^{\circ}$ Composition corporelle.

Le nombre limité des mesures ne permet aucune interprétation statistique; les comparaisons des valeurs de l'épaisseur du lard du cou, du dos, et du rein ont été effectuées entre les groupes de même portée et ont permis leur classification suivant l'adiposité relative :

$$
\begin{aligned}
& e \mathrm{~A}(7)\left({ }^{1}\right)=\text { IOO } \\
& e a(\mathrm{I} 2)=\mathrm{II} 7 \\
& \mathrm{EA}(4)=\mathrm{I} 20 \\
& \mathrm{E} a(7)=\mathrm{I} 25
\end{aligned}
$$

On peut remarquer que celle-ci reflète les valeurs du rapport $\frac{\text { Énergie }}{\text { Protéines }}$ des différents aliments : les animaux sont d'autant plus gras que ce rapport est élevé.

\section{DISCUSSION}

Les résultats des deux expériences peuvent s'interpréter simultanément et s'inscrivent assez bien dans le cadre plus général des relations entre énergie et pro-

(1) Nombre d'animaux. 
téines. 'Toutefois, compte tenu des conditions expérimentales, il est difficile de tirer des conclusions générales de ces résultats, et nous n'insisterons ici que sur les faits essentiels.

$$
\text { Ingérés }
$$

Dans les deux expériences, le résultat le plus remarquable concerne l'ajustement des quantités consommées en fonction des teneurs énergétiques des aliments : toute augmentation du taux d'énergie de l'aliment a pour premier effet une diminution de la quantité d'aliment consommée, de telle sorte que l'énergie ingérée ne varie que dans de faibles limites.

Cette constatation concorde parfaitement avec les observations de nombreux auteurs sur les espèces les plus diverses. C'est ainsi que Hiri, et al. (I954), Phtírson et al. (I954), Lfong et al. (I955), sur le poussin ; StTromingir (I954), Bowi.AND

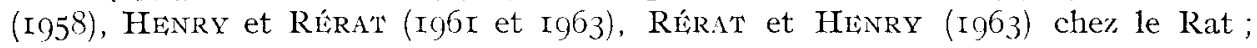
Cirawson et al. (Ig62); Kuryviar, et al. (I062), 'Thrasher et al. (I962) chez le Pore ont montré à des legrés divers que l'animal ingérait, quelle que soit la nature đu régime, des quantités d'énergie sensiblement constantes qui ne dépendaient que du poids de l'animal.

Cette loi très générale subit toutefois quelques exceptions telles que celles rapportées par BowlaNi) (I957), POND (Ig60), ARxouid (I96r) et celle qui apparaît également dans le lot. Fa de notre première expérience. Il n'en reste pas moins que dans la plupart des cas l'enrichissement en énergie des aliments n'apparaît pas comme un facteur important de variation de l'ingéré énergétique (BOWLAND, I958). On peut d'ailleurs se demander dans quelle mesure la présence de taux élevés de lipides dans la ration ne constitue pas un facteur spécifictue qui limite la consommation. Une expérience actuellement en cours semble toutefcis permettre d'écarter cette hypothèse (Aunaitre, résultats non publiés).

\section{Gain de poids}

L'influence des régimes sur le gain de poids des porcelets diffère profondément suivant qu'il s'agit d'animaux sous la mère ou de porcelets sevrés.

Dans le cas du porcelet sous la mère, le gain de poids paraît indépendant de la composition de l'aliment complémentaire et, en particutlier, n'est pas affecté par les variations d'ingérés protidiques qui peuvent apparaitre.

Par contre, chez le porcelat sevré à 5 semaines, l'ingéré protidique semble conditionner le gain de poids et le meilleur régime est celui qui provoque simultanément l'ingestion de protéines et d'énergie les plus grandes.

Ces conclusions sont en parfait accord avec les résultats de Pæo et al. (I957), SEWel, et al. (I96I), Cr.AWSON et al. (I962), Lowriy et al. (I962) sur un matériel analogue.

\section{Efficacité alimentaire}

Les variations d'efficacité alimentaire sont plus délicates à interpréter et diffèrent suivant qu'il s'agit des protéines ou de l'énergie.

Dans les 2 expériences, l'utilisation des protéines semble indépendante à la fois des quantités de protéines et d'énergie ingérées. La seule modification observée est 
liée à l'effet dı taux protidique de l'aliment : l'efficacité protidique diminue légèrement quand le taux azoté augmente, notion parfaitement classique.

En ce qui concerne l'utilisation de l'énergie, les résultats sont différents suivant la technique d'élevage :

ro Chez le porcelet allaité, l'efficacité énergétique reste rigoureusement constante dans tous les lots. Au vu cle ces résultats, il semblerait qu'il y ait intérêt à faire bénéficier l'animal d'un régime complémentaire qui provoque l'ingestion d'énergie la plus grande. Ceci peut s'expliquer d'ailleurs par l'importance quantitative et qualitative de l'apport protidique du lait de la mère qu'un supplément d'énergie alimentaire contribue alors à valoriser pleinement (WEIsCH, Ig63).

$2^{\circ}$ Chez le porcelet sevré à 5 semaines, il en va différemment puisque l'on observe une diminution de l'efficacité énergétique qui paraît liée avant tout à la teneur en énergie de l'aliment. Ceci est en plein accord avec les observations de Crawson et al. (I962) et de LowREY et al. (I962).

Ce résultat s'explique parfaitement par l'augmentation de l'adiposité des animaux avec les aliments riches en énergie, ce qui a déjà été souligné par d'autres auteurs (Kurvviai, et al., I962).

$$
\text { Rapport Energie }
$$

I a notion de rapport $\frac{\text { Énergie }}{\text { Protéines }}$ est familière aux nutritionnistes (Gurr.AUne, I96I) et nous permet de résumer un certain nombre de nos conclusions. Si l'on considère que le meilleur régime est celui qui concilie un gain journalier important et des efficacités protidiques et énergétiques aussi élevées que possible, les conclusions sont différentes suivant qu'il s'agit du porcelet allaité ou du porcelet sevré.

I a figure 2 montre très nettement que pour le porcelet sous la mère le gain de poids et l'efficacité énergétique restent constants ; seule l'efficacité protidique augmente avec le rapport $\frac{\text { Énergie }}{\text { Protéines }}$, le meilleur régime est celui qui présente le rapport le plus élevé.

Par contre, pour le porcelet sevré (fig. 3), lorsque le rapport Énergie $\frac{\text { Éngmente, }}{\text { Protéines }}$ l'efficacité énergétique puis le gain de poids diminuent alors que l'efficacité protidique augmente : un rapport $\frac{\text { Énergie }}{\text { Protéines }}$ faible doit permettre un meilleur résultat d'ensemble pour les trois critères.

On peut alors conclure que la composition des aliments destinés au porcelet doit varier suivant le mode d'élevage : dans nos conditions expérimentales, c'est le régime $\mathrm{E} a$ ( $\mathrm{I} 3 \mathrm{p}$. Ioo de protéines et 4400 Calories brutes par $\mathrm{kg}$ ) qui présente le maximum d'avantages économiques pour le porcelet allaité.

Par contre, c'est le régime Ae (20 p. Ioo de protéines et 4 ooo Calories brutes par $\mathrm{kg}$ ) qui doit être préféré pour le porcelet à 5 semaines. Bien entendu, ceci est valable dans les limites de notre expérience et en particulier pour le mél protidique considéré (CAL,ET et al., I96I). 


\section{SUMMARY}

\section{EFFECT OF ENERGY AND PROTEIN LEVELS IN THE DIET ON EFFICIENCY OF FEED UTILISATION AND GROWTH OF YOUNG PIGS}

Two experiments were undertaken to define the energy and protein levels in feeds for young pigs. The first was with 28 litters suckled from birth to 8 weeks of age and the second was with $\mathrm{x} 2$ litters weaned at 5 weeks of age and studied from then to 8 weeks. In the different trials the piglets were given to appetite a feed as supplement to the milk or a complete feed, in a $2 \times 2$ factorial design :

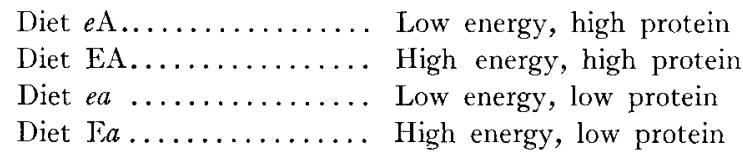

In both the experiments the feed intake was affected by the energy content of the diet but the intake of energy was similar in all groups.

In the suckled pigs growth rate and efficiency of energy utilisation did not differ between diets, and only the efficiency of protein utilisation was affected. The best diet seemed to be that which was poor in protein and rich in energy, I3 p. I00 crude protein and $4400 \mathrm{kcal}$ gross energy per $\mathrm{kg}$.

In the weaned pigs the diets which gave the greatest intake of protein allowed the best growth, but efficiency of energy utilisation was more variable than that of protein. The best diet seemed to be that rich in protein and with medium energy, $20 \mathrm{p}$. I00 crude protein and $4000 \mathrm{kcal}$ gross energy per kg.

\section{RÉFÉRENCES BIBLIOGRAPHIQUES}

ARNould R., I96I. L'utilisation des protéines pour la croissance. Thèse doctoral Sciences agronomiques, Institut agronomique, Université de Louvain.

Aumaitre A., Salmon-Legagneur E., i $96 \mathrm{r}$. Influence de l'alimentation complémentaire sur la croissance du porcelet avant le sevrage. Ann. Zootech., 10, i27-1 40.

Aumaitre A., 1963. Données non publiées.

Bowland J. P., Sibbald I. R., Berg R. T., Hussar N., I958. Influence of dietary fat on energy consumption and digestion and on nitrogen utilization of weanling rats. Canad. J. animal. Sci., 38, i87-193.

Calet C., Jouandet C., Baratou J., I96ז. Variation de la consommation spontanée d'énergie du poussin en fonction de la nature des matières azotées du régime. Ann. Biol. anim. Bioch. Biophys., 1, 5-9.

Clawson A. J., Blumer T. N., Smart W. W. G., Barrick E. R., ig62. Influence of energy-protein ratio on performance and carcass characteriscis of swine. J. animal Sci., 21, 62-68.

Cochran W. G., Cox G. M., ig62. Experimental designs, $2^{*}$ éd., John Wiley and Sons, Inc. Publ.

Dumont B. L., FÉvrier R., i957. Méthode de mesure radiographique de l'épaisseur des tissus adipeux sous-cutanés chez le Porc. Ann. Zoolech., 6, 29-40.

Guillaume J., i96r. Les facteurs de variation du rapport calories/matières azotées totales dans les régimes du poussin. Ann. Zootech., 10, 279-31 I.

Hare J. H., Reynolds W. M., Luther H. G., I956. Relationships of protein productive energy and unidentified growth factors in swine nutrition (Abstr.). J. animal Sci., 15, 1240.

HENRY Y., RÉRAT A., I962. Influence de la qualité et de la quantité des matières azotées ingérées sur la consommation spontanée d'énergie chez le Rat en croissance. Ann. Biol. anim. Bioch. Biophys., 2, $267-276$.

HENRY R., RÉRAT A., I963. Étude de l'ingestion spontanée d'éléments énergétiques et de protéines chez le Rat en croissance par la méthode du libre choix. Ann. Biol. anim. Bioch. Biophys., 3, Io3-I 7.

Hill, F. W, Dansky L. M., I964. Studies on energy requirement of chickens. I. The effect of dietary energy levels on growth and feed consumption. Poult. Sci., 33, I12-i I9.

Kuryvial M. S., Bowland J. P., BERG R. T., I962. Supplemental fat as an energy source in the diets of swine and rats. I.Food and energy utilization. Canad. J. animal Sci., 42, 23-32. 
Leong H. C., Sunde M. L., Bird H. R., Elvehjea C. A., i955. Interelationships among dietary energy, protein and amino acids for chickens. Poult, Sci., 34, I 267-I 284.

LEROY A. M., 1937. Elevage rationnel des animaux domestiques. Hachette éd., Paris.

Leroy A. M., I954. Utilisation de l'énergie des aliments. Ann. Zootech, 3, 337-372.

Lowrey R. S., PoNd W. G., Loosli J. K., Barnes R. H., Ig63. Effect of dietary protein and fat on growth, protein utilization and carcass composition of pigs fed purified diets. J. animal Sci., 22, Iog-I I4.

Mollgardo H, Futterungslehre des Milchviehs. Schäper ed., Hanover, I929.

PÉo E. R., Ashton G. C., Speer N. C., CAtron D. V., 1957. Protein and fat requirements of baby pigs. J. animal Sci, 16, 885-89r.

Peterson D. W., Grau C. R., Peek N. F., I954. Growth and food consumption in relation to dietary levels of protein and fibrous bulk. J. Nutr., 52, $241-257$.

Poley G. E., SEerley R. W., I96r. Energy-protein relationship in rations for growing finishing swine (Abstr.). J. animal Sci., 20, 946-947.

Pond W. G., Kwong E., Loosli J. K., i 1960 . Effect of level of dietary fat, panthotenic acid and protein on performance of growing fattening swine. J. animal Sci., 19, II I 5-I I22.

RÉrat A., HENRY Y., I963. Étude de l'ajustement de la consommation spontanée d'énergie en fonction de l'apport azoté chez le Rat en croissance. Ann. Biol. anim. Bioch. Biophys., 3, 263-298.

Satmon-Legagnelr E., 1959. La composition du lait de Truie. Premières observations sur quelques facteurs de variation. Ann. Zootech., 8, 93-I I2.

Salmox-Legagneur E., Atmaitre A., I962. Influence de la quantité de lait et de sa composition sur la croissance du porcelet sous la mère. Ann. Zootech., 11, 181-196.

Salmon-Legagneur E., Jolandet C., 1962 . Note sur le sevragre des porcelets à l'âge de 5 semaines. Ann. Zootech., 11, 3I I-313.

Sewell R. F., Thomas II. C., PRICE I). I96I. Protein-energy relationship in the rations of early weaned pigs, J. animal Sci., 20, 820-823.

Stroninger J. L., Brobeck J. R., CORT R. L., I958. Regulation of food intake in normal rats and in rats with hypothalamic hyperphagia. Yale $]$. Biol. Med., 26, 55-74.

Thrasher D. M., Mullins A. M., Newman C. W., Bovlware R. F., Hansard S. L., ig6z. Effect of level of protein and fat on performance and carcass merit of growing finishing swine (Abstr.). J.animal Sci., 21, 377.

Wagner G. R., Clark A. J., Hays V. W., Speer V. C., McCall J. T., I96I. Effect of energy and protein intake and sex on growth and feed efficiency of pigs (Abstr.). J. animal Sci., 20, 955.

Welsch J. G., Vander Noot G. W., Gilbreath R. L., ig63. Effect of feeding milk replacers with varying amounts of fat for hothouse lamb production. J.animal Sci., 22, ${ }_{15} 5^{-1} 58$. 